\title{
Magnetic fields of weak line T-Tauri stars
}

\author{
Colin A. Hill and the MaTYSSE Collaboration \\ Université de Toulouse / CNRS-INSU, IRAP / UMR 5277, F-31400, Toulouse, France \\ email: chill@irap.omp.eu
}

\begin{abstract}
T-Tauri stars (TTS) are late-type pre-main-sequence (PMS) stars that are gravitationally contracting towards the MS. Those that possess a massive accretion disc are known as classical T-Tauri stars (cTTSs), and those that have exhausted the gas in their inner discs are known as weak-line T-Tauri stars (wTTSs). Magnetic fields largely dictate the angular momentum evolution of TTS and can affect the formation and migration of planets. Thus, characterizing their magnetic fields is critical for testing and developing stellar dynamo models, and trialling scenarios currently invoked to explain low-mass star and planet formation. The MaTYSSE programme (Magnetic Topologies of Young Stars and the Survival of close-in Exoplanets) aims to determine the magnetic topologies of $\sim 30$ wTTSs and monitor the long-term topology variability of $\sim 5$ cTTSs. We present several wTTSs that have been magnetically mapped thus far (using Zeeman Doppler Imaging), where we find a much wider range of field topologies compared to cTTSs and MS dwarfs with similar internal structures.
\end{abstract}

Keywords. stars: magnetic fields, stars: pre-main-sequence, stars: spots, stars: late-type, stars: activity, stars: imaging, techniques: polarimetric, techniques: radial velocities

\section{Introduction}

T-Tauri stars (TTS) are young, low mass pre-main-sequence (PMS) stars that are equivalent to young Suns. After emerging from their dust cocoons, newly formed protostars are initially surrounded by a large-scale magnetized accretion disc. These protostars eventually settle as gravitationally contracting PMS stars surrounded by a protoplanetary disc (André et al. 2009), and at an age of 0.5-10 Myr, they are known as classical T-Tauri stars (cTTSs) if they are still possess a massive (presumably planet forming) accretion disc, or weak-line T-Tauri stars (wTTSs) if they have exhausted the gas in their inner discs. Both cTTSs and wTTSs are excellent test beds for theories of low-mass star and planet formation, and a such, have been studied intensely over the last few decades.

Magnetic fields in such objects have their largest impact during the star's early evolution, with fields controlling accretion processes and triggering outflows, as well as largely dictating the angular momentum evolution of low-mass PMS stars (e.g. Bouvier et al. 2007, Frank 2014). In particular, large-scale fields of cTTSs can evacuate the central regions of accretion discs, funnel the material onto the star, and enforce co-rotation between the star and the inner disc Keplerian flows, causing cTTSs to rotate more slowly than expected from the contraction and accretion of the disc material (e.g. Davies et al. 2014). Furthermore, magnetic fields of cTTS and their discs can affect the formation and migration of planets (e.g. Baruteau et al. 2014). Moreover, fields of both cTTSs and wTTSs are known to trigger thermally driven winds through heating by accretion shocks and/or Alfvén waves (e.g. Cranmer 2009, Cranmer \& Saar 2011), resulting in flares, coronal-mass ejections, and angular momentum loss (e.g. Aarnio et al. 2012, Matt et al. 2012).

The importance of magnetic fields in both the long and short-term evolution of TTS is clear, and in order to develop and test theoretical models to provide more physical 
realism and reliable predictions, it is crucial that we characterize the magnetic fields in cTTSs and wTTSs through observations.

\section{Observing magnetic fields of T-Tauri stars}

Despite first detecting magnetic fields on cTTSs nearly 20 years ago (e.g. John-Skrull et al. 1999), their large-scale topologies have only recently been revealed for a dozen stars through the MaPP (Magnetic Protostars and Planets) programme (e.g. Donati et al. 2007, 2010, 2013, Hussain et al. 2009). The MaPP survey revealed that the largescale fields of cTTSs remain relatively simple and mainly poloidal when the host star is still fully or largely convective, but become much more complex when the host star turns mostly radiative (Gregory et al. 2012, Donati et al. 2013). This survey also showed that these fields are likely of dynamo origin, varying over timescales of a few years (Donati et al. 2011, 2012, 2013), and resembling those of mature stars with comparable internal structure (Morin et al. 2008).

By contrast, only a few wTTS have been magnetically imaged (and published) to date, namely V410 Tau, LkCa 4, V819 Tau, V830 Tau and TAP 26 (Skelly et al. 2010, Donati et al. 2014, 2015, Yu et al. 2017). These few stars show a much wider range of field topologies compared to cTTSs and MS dwarfs with similar internal structures; while V819 Tau and V830 Tau display a mostly poloidal field topology, V410 Tau and LkCa 4 show significant toroidal components despite being fully convective, in surprising contrast to fully convective cTTSs and mature M dwarfs that harbour relatively simple poloidal fields (Morin et al. 2008, Donati et al. 2013).

Studies of magnetic fields (and associated winds) on wTTSs are of great interest, as these are the initial conditions in which disc-less PMS stars initiate their unleashed spinup as they contract towards the MS. This is one of the goals of the MaTYSSE (Magnetic Topologies of Young Stars and the Survival of close-in Exoplanets) Large Programme, allocated at the 3.6-m CFHT at Mauna Kea on Hawaii, using the ESPaDOnS spectropolarimeter over semesters $2013 \mathrm{a}-2016 \mathrm{~b}(510 \mathrm{~h})$, with complementary observations using NARVAL on the 2-m TBL at Pic du Midi in France $(420 \mathrm{~h})$, and using the HARPS spectropolarimeter at the 3.6-m ESO Telescope at La Silla in Chile (70 h).

MaTYSSE has several aims; Firstly, to determine the magnetic topologies of $\sim 30$ wTTSs and compare them to those of cTTSs to discover if their large-scale fields are similar, or significantly different. In particular, as wTTSs are no longer accreting, we may assess the role of accretion and the impact of its interruption, given that accretion or star/disc coupling torques may modify dynamo processes and the corresponding large-scale field topology. Furthermore, we can study magnetic winds of wTTSs and corresponding spin-down rates (e.g. Vidotto et al. 2014), painting a picture of the kind of magnetospheres that young Sun-like stars have when they contract and spin-up towards the MS, thus consistently explaining the rotational history of low-mass stars once on the MS (given that magnetic braking is the main cause of their spin down). Moreover, we will monitor the long-term magnetic topology variability of $\sim 5$ cTTSs, and study the variability of magnetospheric gaps and winds due to non-stationary dynamos, aiming to determine if disc migration is the main process for producing hot Jupiters (hJs), and if magnetospheric gaps and winds key are factors for their survival. If this is the case, we can expect to find at least as many hJs in TTSs as are in mature stars, and significantly more if we account for those absorbed by protostar over the contraction phase.

To map surface features (such as spots and plages) and magnetic fields on our target stars, we use our Zeeman Doppler Imaging (ZDI) code that has been specifically adapted for use with wTTSs. First, we take our Stokes $I$ and $V$ spectra and use Least-Squares 

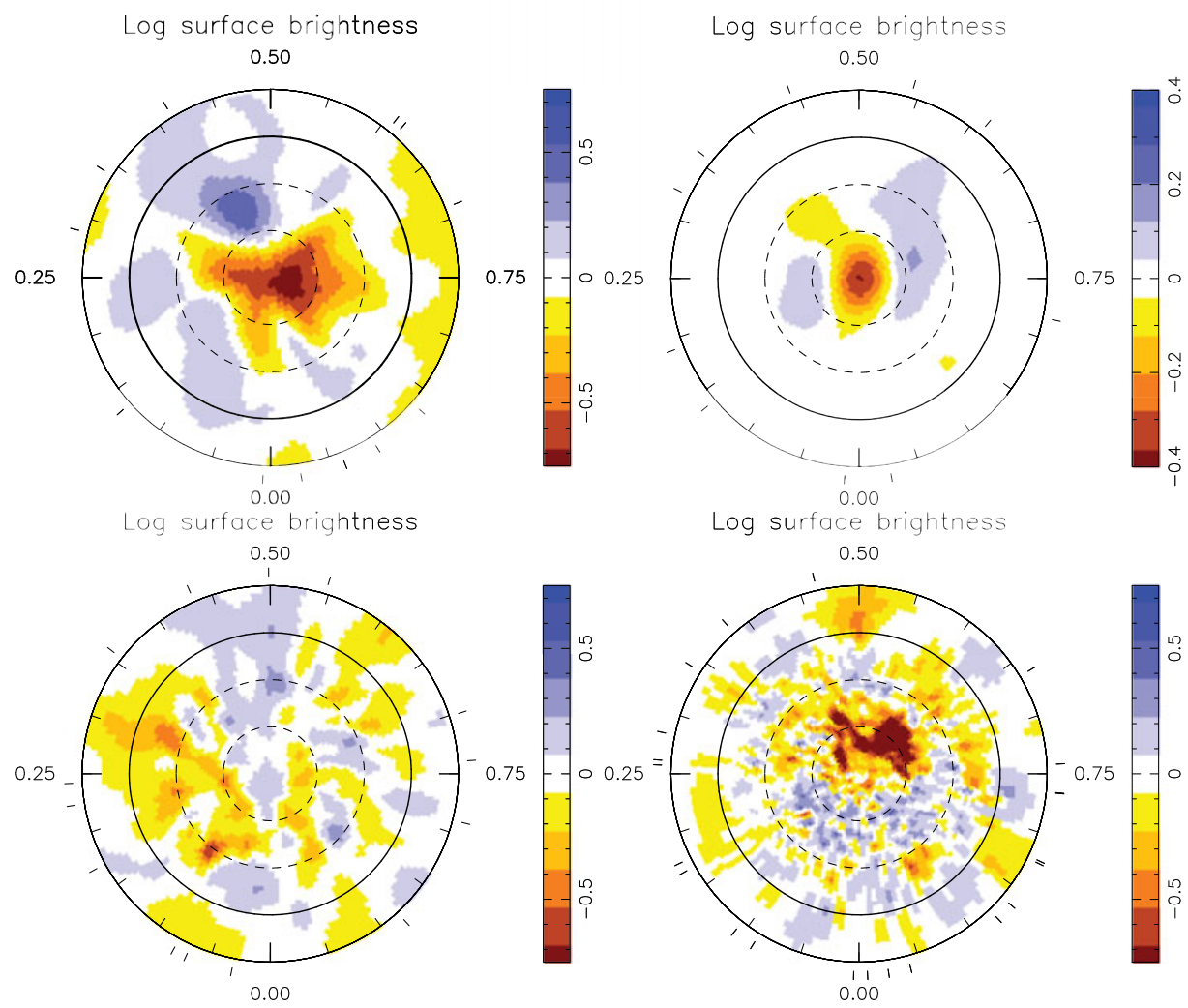

Figure 1. Maps of the logarithmic brightness (relative to the quiet photosphere), at the surfaces of LkCa 4 (top left), Par 1379 (top right), Par 2244 (bottom left) and TWA 6 (bottom left). The stars are shown in flattened polar projection down to latitudes of $-30^{\circ}$, with the equator depicted as a bold circle, and $30^{\circ}$ and $60^{\circ}$ parallels as dashed circles. Radial ticks around each plot indicate phases of observations. This figure is best viewed in colour.

Deconvolution (LSD) to create a 'mean' line profile (with a greatly increased S/N, see Donati et al. 1997). Then, we simultaneously invert a time series of these Stokes $I$ and $V$ LSD profiles (using a spherical harmonic decomposition for the magnetic field, and using maximum-entropy regularization) to create our reconstructed maps (see Figures 1 and 2).

As well as determining the magnetic field topology, we can use the brightness maps from ZDI to predict the activity-related radial velocity (RV) jitter due to spots and/or plages. We can then filter the RV measurements to detect potential hot Jupiters (hJs) around wTTSs, and thus verify whether core accretion and migration is the most likely mechanism for forming close-in giant planets (see Chapter ??? by $\mathrm{Yu}$ in this volume; Donati et al. 2016).

\section{Results}

Figures 1 and 2 show the brightness and magnetic maps of several wTTSs from the MaTYSSE sample. These few stars (namely, LkCa 4, Par 1379, Par 2244 and Twa 6) demonstrate the wide range of magnetic topologies that can exist in this class of $\mathrm{T}$ Tauri star. In particular, LkCa $4\left(0.9 \mathrm{M}_{\odot}, 2 \mathrm{Myr}, v \sin i=28 \mathrm{kms}^{-1}, P_{\text {rot }}=3.37 \mathrm{~d}\right)$ 

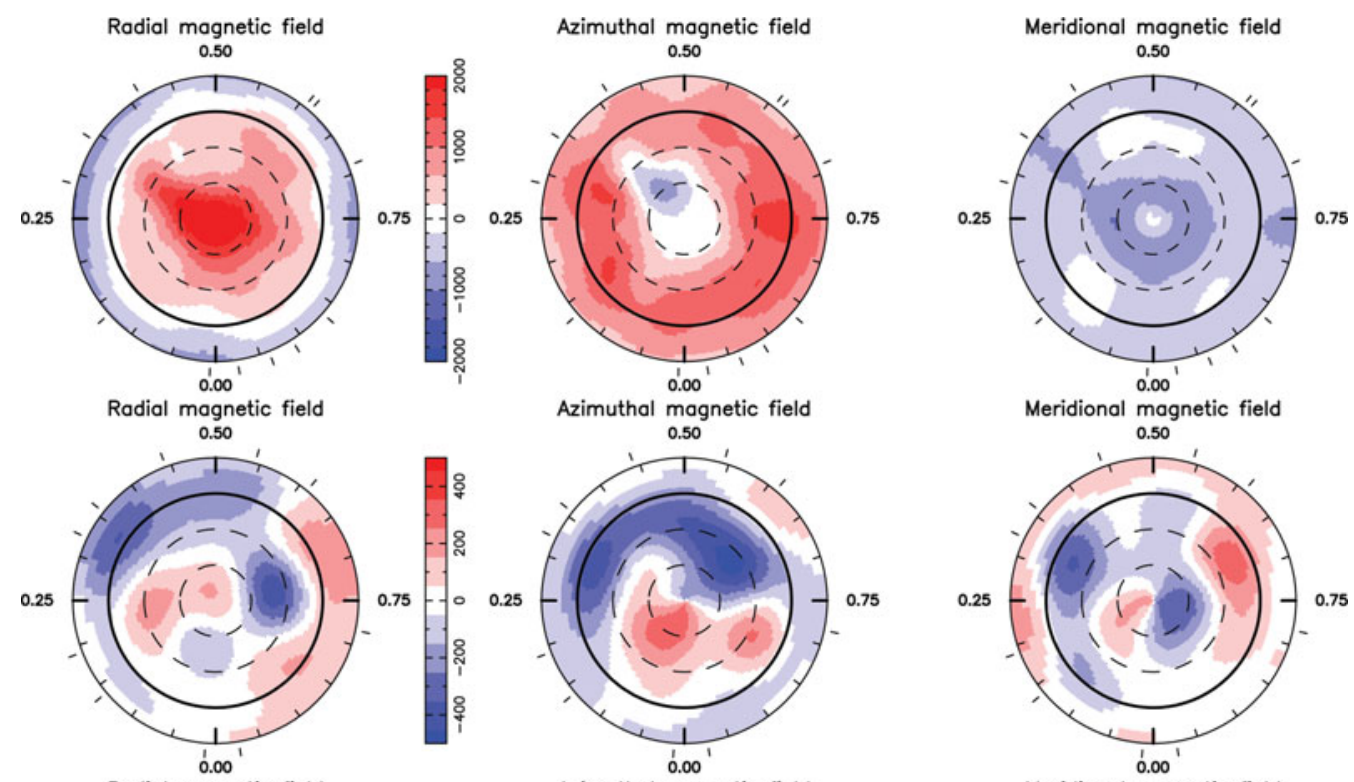

Azimuthal magnetic fie

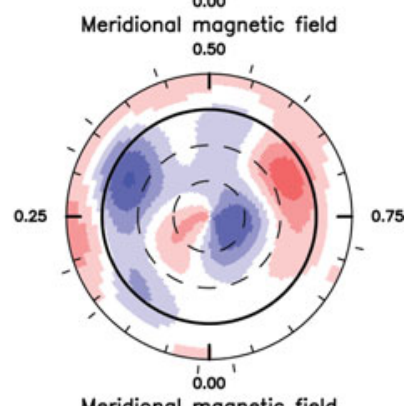

Radial magnetic field

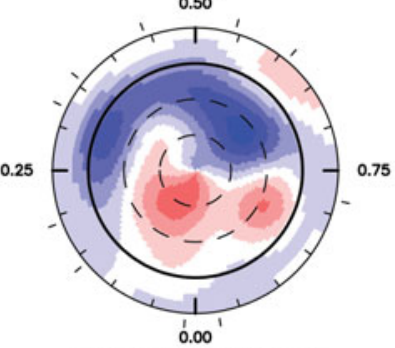

Azimuthal magnetic field
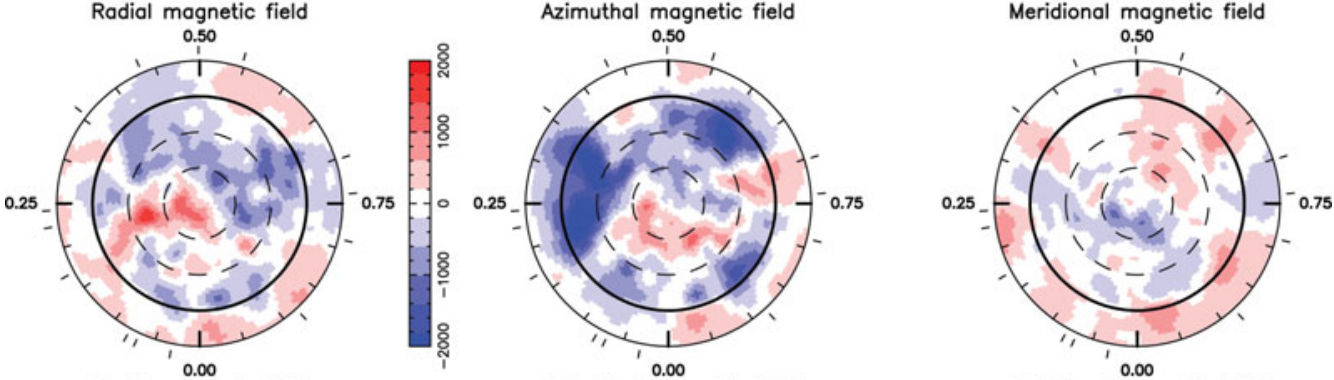

Radial magnetic field
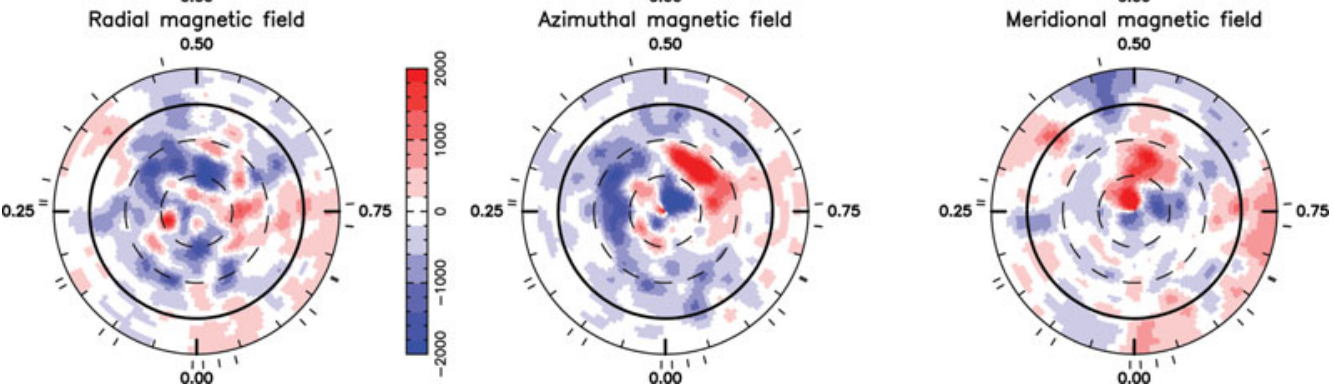

Figure 2. Maps of the radial (left column), azimuthal (middle column) and meridional (right column) components of the magnetic field $\mathbf{B}$ at the surfaces of (top to bottom) LkCa 4, Par 1379, Par 2244 and TWA 6. Magnetic fluxes in the colourbar are expressed in G. The stars are shown in flattened polar projection as in Figure ??. This figure is best viewed in colour.

shows a simple, strong and mainly axisymmetric field featuring a $2 \mathrm{kG}$ aligned-poloidal component and a $1 \mathrm{kG}$ toroidal component encircling the star at equatorial latitudes (with the latter feature markedly different from cTTSs of similar mass and age). In contrast, Par $1379\left(1.6 \mathrm{M}_{\odot}, 1.6 \mathrm{Myr}, v \sin i=13.7 \mathrm{kms}^{-1}, P_{\text {rot }}=5.585 \mathrm{~d}\right)$ shows a much weaker, mostly non-axisymmetric field, with a $75 \mathrm{G}$ aligned-poloidal component and a $400 \mathrm{G}$ toroidal field. The similarly massive wTTS, Par $2244\left(1.8 \mathrm{M}_{\odot}, 1 \mathrm{Myr}\right.$, $v \sin i=57.2 \mathrm{kms}^{-1}, P_{\text {rot }}=2.8153 \mathrm{~d}$ ), features a much more complex field topology, with a mostly non-axisymmetric aligned-poloidal component of $800 \mathrm{G}$, and a mostly 


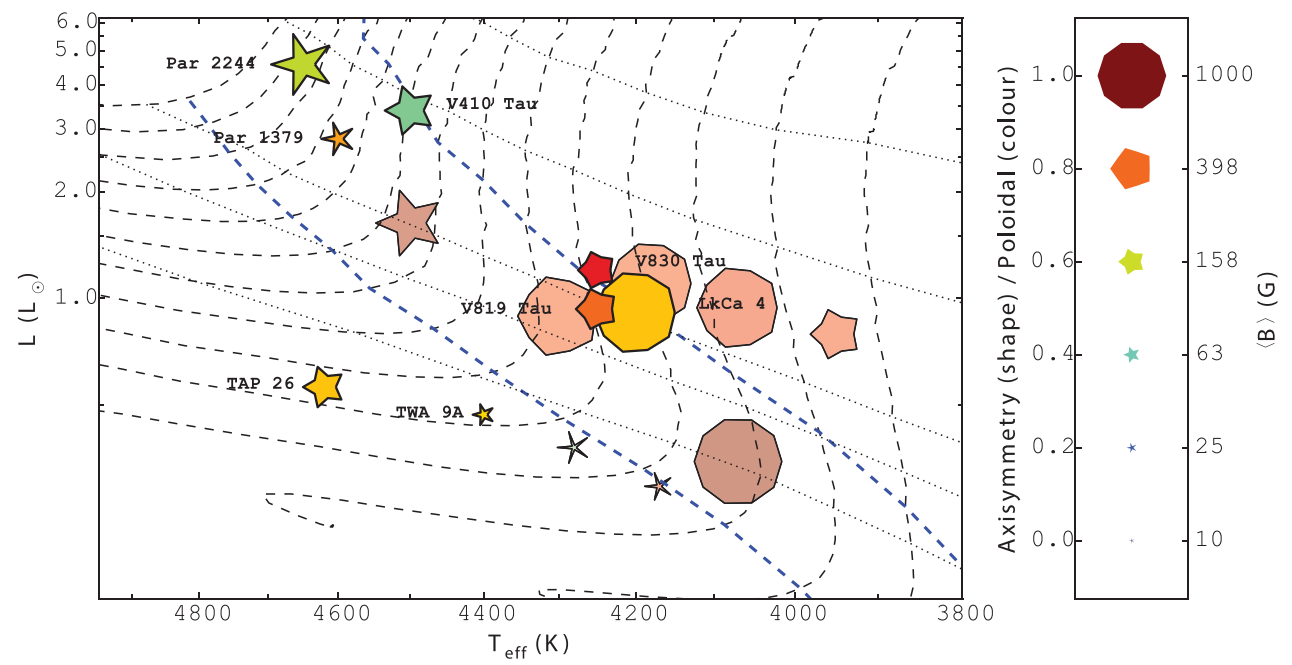

Figure 3. H-R diagram showing the MaTYSSE wTTSs (solid colours) and the MaPP cTTs (translucent/faded colours). The size of the symbols represents the surface-averaged magnetic field strength (with a larger symbol meaning a stronger field), the colour of the symbol represents the fraction of the field that is poloidal (with red being completely poloidal), and the shape of the symbols represents the axisymetry of the poloidal field component (with higher axisymmetry shown as a more circular symbol). Also shown are evolutionary tracks from Siess 2000 (black dashed lines, ranging from $0.5-1.9 \mathrm{M}_{\odot}$ ), with corresponding isochrones (black dotted lines, for ages of $0.5,1,3,5 \& 10 \mathrm{Myr}$ ), and lines showing $0 \%$ and $50 \%$ convective by radius (blue dashed).

axisymmetric toroidal component, with fields in excess of $2 \mathrm{kG}$. Finally, the much older wTTS, Twa $6\left(0.9 \mathrm{M}_{\odot}, 20 \mathrm{Myr}, v \sin i=72.6 \mathrm{kms}^{-1}, P_{\text {rot }}=0.5409 \mathrm{~d}\right)$, harbours a mostly axisymmetric field with a $900 \mathrm{G}$ aligned-poloidal field and a toroidal component with surface fields in excess of $2 \mathrm{kG}$.

To put these stars in context, we have plotted in Figure 3 an H-R diagram of the cTTSs from the MaPP programme, as well as the (analysed) MaTYSSE stars. Figure 3 also indicates the fraction of the field that is poloidal, the axisymmetry of the poloidal component, and shows PMS evolutionary tracks from Siess et al. 2000. So far, our sample of wTTSs show a much wider range of field topologies compared to cTTSs. We find that large scale fields can be more toroidal and non-axisymmetric than cTTSs, with a significant toroidal component emerging after disc dissipation.

This preliminary result demonstrates the eclectic mix of magnetic field topologies in wTTSs, and once a complete analysis is made of the MaTYSSE sample, we will be able to robustly compare the magnetic topologies of cTTSs and wTTSs, assessing the role of accretion and the presence of a disc on dynamo processes. Furthermore, we will determine the nature of the magnetic winds on wTTSs, and the frequency of hJs, leading to a better understand their formation and migration.

\section{References}

Aarnio, A. N., Matt, S. P., \& Stassun, K. G., 2012, ApJ, 760, 9

André, P., Basu, S., \& Inutsuka, S., 2009, The formation and evolution of pre-stellar cores. Cambridge University Press, p. 254

Baruteau, C., et al., 2014, Protostars and Planets VI, pp 667-689

Bouvier, J., Alencar, S. H. P., Harries, T. J., Johns-Krull, C. M., \& Romanova, M. M., 2007, Protostars and Planets $V$, pp 479-494 
Cranmer, S. R., 2009, ApJ, 706, 824

Cranmer, S. R. \& Saar, S. H., 2011, ApJ, 741, 54

Davies, C. L., Gregory, S. G., \& Greaves, J. S., 2014, MNRAS, 444, 1157

Donati, J.-F. et al., 1997, MNRAS, 291, 658

Donati, J.-F. et al., 2007, MNRAS, 380, 1297

Donati, J.-F. et al., 2010, MNRAS, 402, 1426

Donati, J.-F. et al., 2011, MNRAS, 412, 2454

Donati, J.-F. et al., 2012, MNRAS, 425, 2948

Donati, J.-F. et al., 2013, MNRAS, 436, 881

Donati, J.-F. et al., 2014, MNRAS, 444, 3220

Donati, J.-F. et al., 2015, MNRAS, 453, 3706

Donati, J.-F. et al., 2016, Nature, 534, 662

Gregory, S. G., Donati, J.-F., Morin, J., Hussain, G. A. J., Mayne, N. J., Hillenbrand, L. A., \& Jardine, M., 2012, ApJ, 755, 97

Hussain, G. A. J., et al., 2009, MNRAS, 398, 189

Johns-Krull, C. M., Valenti, J. A., \& Koresko, C., 1999, ApJ, 516, 900

Matt, S P., 2012, ApJ, 745, 101

Morin, J., et al., 2008, MNRAS, 390, 567

Siess, L., et al., 2000, A\&A, 358, 593

Skelly, M. B., Unruh, Y. C., Collier Cameron, A., Barnes, J. R., Donati, J.-F., Lawson, W. A., \& Carter, B. D., 2008, MNRAS, 385, 708 